\title{
THE MENTAL TELESCOPE: UNDERSTANDING THE GEOMETRY OF EUCLID BY LEARNING THE NON-EUCLIDEAN GEOMETRY
}

\author{
S. Benvenuti ${ }^{1}$, A. Cardinali ${ }^{2}$ \\ ${ }^{1}$ Università di Camerino (ITALY) \\ ${ }^{2}$ Scuola di Scienze e Tecnologie - Sezione di Matematica - Università di Camerino (ITALY)
}

\begin{abstract}
We describe a research project about the teaching of non-Euclidean geometries. Our intent is to show that non-Euclidean geometries can be a tool to promote the understanding of the modern axiomatic method in mathematics, to stimulate students' aptitudes to logical thinking and to allow students to consolidate the knowledge of Euclidean geometry by developing it in a critical way.
\end{abstract}

Keywords: Non-Euclidean geometries, Axiomatic method.

\section{STATE OF THE ART}

Education in Italy is overseen by MIUR (Ministero per l'Istruzione, l'Università e la Ricerca), a ministry responsible for compulsory school and higher level education, university and academic research. All levels of compulsory school have teaching recommendation or guidelines for each subject that are suggested by the MIUR. For each subject the teachers discuss those recommendation, plan activities and each teacher can further adapt its teaching program for a specific class. Mathematics is arguably one of the core subjects in the lyceum ("Liceo") high school type. It provides the student, together with more human-related subjects such as Phylosophy, Physics, Literature and Latin a broad vision of past and present human knowledge. Specifically, with sub-topics as Geometry, Calculus, Arithmetic, etc., it provides an analytic means to shape the student's ability to understand scientific knowledge, the progress of scientific thinking and all the tools to develop a mind open to scientific studies if enrolled in a university program.

Current lyceum high school national recommendations, "Indicazioni Nazionali" [1] introduced in 2010 with the so-called Gelmini-Reform (reported in [2]) - reform named by the Italian Minister of Education Mariastella Gelmini - suggest that the student should be able - at the end of his/her studies - to understand the historical context of several mathematical theories and their conceptual meaning. The recommendation also suggest "a clear vision of the axiomatic approach in its modern conception and of its specificity with respect to the classic Euclidean approach". Nonetheless, the recommendation do not report non-Euclidean geometries among the suggested teaching topics. As the italian mathematician and teacher Walter Maraschini (1949-2017) critically stated in a talk during the round table of the XXIX UMI Conference, this lack weakens the coherence of the recommendation [3]. In the contemporary historical and methodological asset of mathematical studies, it is hard to understand the modern axiomatic approach without studying non-Euclidean geometries, which differently from Euclidean "descriptive" method, is a hypothetic-deductive method. Indeed, the failure of an absolute model of space as a groundtruth drives modern efforts to define a new mathematical theory based on axioms. "Non-Euclidean Geometry is not only true, but also necessary. Without it, the development of so-called modern mathematics would be hardly conceivable" [4].

The rise of non-Euclidean geometries and the demonstration of their logical consistency, guaranteed by the models conceived by Beltrami, Klein and Poincarè, represented a fundamental turning point in the development of scientific thinking. Recalling the words of Imre Toth [5], the great novelty has been the establishment of a plurality of worlds: the universe of geometry is no longer a domain in which there is only one valid truth, two opposing truths are valid for the subject. The problem of guaranteeing the consistency of a system of axioms chosen through an act of freedom and without the claim to base their validity on some "evidence" becomes of fundamental importance. By excluding non-Euclidean geometries from the teaching programmes, the recommendation exclude, thus, a large part of the mathematical issues from the $20^{\text {th }}$ century, showing again a contradiction because at the same time they suggest to report results from $20^{\text {th }}$ century mathematical and scientific results in Phylosophy courses. To mitigate the importance of some of the lacks and the contradiction hereby discussed, the following quote from [1] is reported: "these recommendation do not dictate any didactical or pedagogical model. [...] The teacher has freedom to enrich what is suggested by these 
recommendation, with respect to the characteristics of each lyceum curriculum, and also has the freedom to apply the appropriate strategies and methods."

Before the so-called Gelmini reform, many Italian high schools were experimenting the PNI plan (Piano Nazionale Informatica) [6]. Among the contents for Geometry courses of the $5^{\text {th }}$ grade of high school, the PNI reported the following contents:

- non-Euclidean geometries from a basic standpoint;

- hypotetic-deductive method: foundations, axioms, definitions, theorems. Coherence and independence of an axiom system;

- the Euclidean geometry as an axiomatic system.

The PNI also commented on this: "Presenting non-Euclidean geometries should not end in itself, but will clarify the meaning of axiom and of a hypothetic-deductive system. [...] Limiting itself to fundamental facts, the teacher will be able to show the most significant attempts to demonstrate the $5^{\text {th }}$ postulate of Euclid and illustrate basic properties of non-Euclidean geometries by comparing them with Euclidean geometry. [...] Critical thinking will bring the student to conclude his high school studies after studying the Euclidean geometry as an axiomatic system."

In Italy, to the best of our knowledge, there have been only a few attempts at introducing nonEuclidean geometry in high school to reinforce axiomatic thinking [7][8][9], although much educational material is available for the non-expert such as books, texts and hypertexts [10], [11], [12], [13]. Among the reported references only [8] reports results regarding the efficacy of the proposed approach, but the work is only aimed at teaching an Euclidean axiomatic formalization. Furthermore, all of these were proposed during the PNI experimental plans, i.e. before Gelmini reform. In the frame of the national PLS project (Progetto Lauree Scientifiche), many laboratories focused on nonEuclidean geometries have been conducted in different university locations, by university referents who collaborate with high school teachers of mathematics. Since 2007, for instance, such laboratories have been organized by the University of Camerino under the supervision of our research team, who works with several high schools in the Marche Region.

Similar issues have also been exposed and discussed in other countries. A few international research papers have been found so far that highlight interesting methods for teaching non-Euclidean geometries. Specifically, in 1993 a research group reports the exploration of hyperbolic geometries employing software tools [14]. A very recent book, Chapter in Mathematics and Technology [15], reports on progresses in teaching hyperbolic geometries by means of the same software of [14] and modern pedagogical resources such as the Drama in Education method. In [16] an experimental program is reported to improve teachers' understanding of Euclid's geometry by confronting them with Taxicab and Spherical geometries. In the 3-weeks course dedicated to primary and secondary (K-12) school, the teachers are forced to rethink their prior knowledge of geometry and apply mathematical reasoning to improve their teaching of Euclidean geometry.

After evaluating all these findings we believe that teaching non-Euclidean geometries in high schools at the moment is overlooked but, even more important, there has never been sufficient research on the efficacy of its teaching.

\section{FINDINGS FROM THE CAMERINO PLS' EXPERIENCE}

Too often mathematics is perceived as an arid and cold discipline, light years away from everyday reality, linked to results established in the most remote antiquity, which can not be improved or refuted. Teaching non-Euclidean geometries can be useful to fight such an impression. It is in fact a mathematics that has been developed in relatively recent years, say around the XVIII century, in a historical period that our students are used to studying and learning, and that they obviously feel closer than it is, for example, 300 a.C. when Euclid formalized in his Elements the mathematical knowledge accumulated until then. Moreover, the natural interdisciplinarity of non-Euclidean geometry, which has deep echoes in art, literature and the interpretation of the physical world, makes it an ideal subject, congenial to both a student who loves science and a one with a more humanistic or artistic taste. Finally, it is often difficult to explain what it means to do research in mathematics: we are deeply convinced, on the basis of our didactic experience, that the example of non-Euclidean geometry, counterintuitive, surprising, and above all alive, makes it immediately understandable. 
On the other hand, it is a fact that (Italian) high school students must necessarily deal with the problem of Euclidean geometry crisis in their course of study. In fact, the proposals of the mathematics programs for lyceum high school foresee, for almost all the addresses, the non-Euclidean geometries from an elementary point of view. Furthermore, philosophy programs include topics such as the second scientific revolution, the new epistemology, mathematics and logic in the nineteenth and twentieth centuries. Faced with this request, the Italian publishing scene is particularly poor: alongside the relative abundance of references available on the web, there are very few books that treat both the historical and the technical aspect of non-Euclidean geometries, rigorously but in an elementary way. Furthermore, scholastic literature totally ignores the echoes of non-Euclidean geometry in art, literature, and the interpretation of the world. Teachers are therefore often in difficulty in dealing with this topic, not only because of the chronic lack of time, but also because of the equally unfortunate lack of available material.

It is starting from this awareness that, since the academic year 2007, our research group has decided to include a laboratory on non-Euclidean geometry among the activities proposed in the framework of the PLS. The idea was providing a useful support for both teachers and students, who actually responded with great enthusiasm to our teaching proposal.

The schools involved, all located in the Marche region, are the scientific lyceum of Camerino, Civitanova Marche, Jesi, Macerata, Recanati, San Benedetto del Tronto and Tolentino. Generally the fourth and fifth classes of these institutes participate in the proposed activities. The following Table 1 shows the numbers related to the laboratories, each to be understood per year, on average.

Table 1. Camerino Pls' laboratories on non-Euclidean geometry.

\begin{tabular}{|l|c|}
\hline Number of students & 200 \\
\hline Number of high school teachers & 12 \\
\hline Number of University teachers & 7 \\
\hline Number of high schools involved & 7 \\
\hline Ratio students/teachers & 16,6 \\
\hline Ratio students/university teachers & 200 \\
\hline Ratio school/university teachers & 12 \\
\hline Total number of hours for each student & 20 \\
\hline Number of hours for designing the laboratory & 10 \\
\hline Number of hours devoted to the autoevaluation & 10 \\
\hline
\end{tabular}

During our laboratories the students are guided to think critically, discovering a different mathematics from the usual one, hidden in unusual and surprising objects and situations. They are constantly encouraged to develop a creative approach to topics and tools that are normally presented in a formal way, which translates into an undoubted cultural enrichment. They are encouraged to create models (physical or virtual) of the mathematical entities with which they come into contact, inspired by those presented and illustrated by the university professor responsible during the introductory lecture.

The laboratory is, in our opinion, an opportunity for professional growth for the teachers involved, as they are able to deal with non-curricular topics, on which it is difficult to find otherwise simple and scientifically correct material. The activities of the laboratories are carried out according to the following procedure: the laboratory opens with an introductory lecture by the university professor of reference; follows an in-depth study in the classroom, supervised by the teacher, who agrees and organizes it with the help of the university teacher and any trained students; a final evaluation of the results is foreseen, which takes place through the analysis of questionnaires elaborated by the school teacher in collaboration with the university teacher.

The proposed activity proved to be very successful, so to lead us to broaden both the number of involved schools and the number and age of the students. Another encouraging sign is the loyalty of the schools involved, which from the beginning of our teaching experience repeat the workshop from year to year. 


\section{PROPOSED RESEARCH}

Following from the related works and the discussion reported above, our goal is to develop a method of teaching that pursues and broadens the orientations and goals expressed by the PNI plan [6]. Specifically, we aim to show whether or not the teaching of non-Euclidean geometries is a useful tool to reinforce learning of the axiomatic method. We believe that an educational strategy is required that highlights the theoretical essence of mathematics without only reducing to a repetitive execution of exercises to be solved. Such educational strategy should be able to reconstruct an ensemble vision and a historical perspective.

The research work will make use of valuable results from other research efforts, such as those presented in the previous section. In particular, the proposed project could merge with the PLS project in order to fruitfully gather previous results and expand the research work, specifically on the evaluation of the methods.

The research plan foreseen follows different phases:

- the first year will be devoted to bibliographic research regarding methods of teaching nonEuclidean geometries and compiling a method for teaching that will be later tested. Several factors must be considered for that extent: the use of software tools and other computer aids, which non-Euclidean geometry to teach and how, duration and periodicity of the lectures. In doing this, international papers of interest will be taken as a reference and adapted to the Italian high school recommendation and best practices.

- Once an experimental plan has been compiled, contacts will be made with high school teachers to propose a collaboration. Not only lyceum type of schools will be addressed in this phase, to broaden the scope of this research and compare different type of schools (e.g. industrial, technical, etc.). Mainly $4^{\text {th }}$ and $5^{\text {th }}$ grade students will be addressed.

- A pilot study is planned for the second year, to test the compiled method. Feedback from the teachers will be valuable in this phase to make adjustment to the method.

- A second round of experiments will follow in the third year with all the adjustments and changes retained important at this time. Results will be gathered and compared with the first round. We should be able to report data and guidelines to be presented to the scientific community.

For what concerns the experimental phase, the following steps can be envisioned:

- preliminary meetings will be organized with the agreeing teachers to present the research activity.

- The starting level of the students will be assessed by competence tests.

- Activities will follow two different modalities: autonomously by the teachers during their classes and following seminars with the University staff. In both cases, it is required that all the students participate, not only the most brilliant ones. Many students, indeed, do not perceive themselves as brilliant in mathematics, however they may still have good reasoning capabilities and benefit from this practice.

- At the end, a final evaluation will be performed to gather data that will be analyzed to evaluate the efficacy of the proposed method.

\section{EXPECTED OUTCOMES}

The proposed research aims at formalizing a teaching method that can be shared with the national and international educational community by means of discussion and dissemination employing the appropriate means such as scientific papers, presentations at educational conferences and debates during round tables. The method should mitigate the lack present in the current national recommendation and could serve as a reference for other countries where the educational community feels a similar need. The goal of this method is to reinforce the student's attitude to logical thinking, and to provide an understanding of the axiomatic formalization required to any mathematical theory in order to be coherent. It is also expected that this method reinforces the knowledge of Euclidean geometry.

These goals will be formalized during the research process so that they can be evaluated quantitatively. Indicators will be derived to evaluate, e.g.: 
- the comprehension of $\bigvee$ Euclid's postulate and equivalent formulations;

- the comprehension of the axiomatic systematization;

- the comprehension of the meaning of non-contradiction in an axiomatic system;

- the comprehension of the meaning of independence of an axiomatic system, i.e. of a postulate from the others;

- the comprehension of building an axiomatic system;

- the ability to understand differences and analogies between different axiomatic systems and models of a same system;

- the knowledge of non-Euclidean geometries (hyperbolic and elliptic) and the ability to define properties and to analyze differences from Euclid's geometry.

Finally, it is expected to give the students a new perspective on mathematics, so that they can see it as a creative activity, a debatable topic with a historical progression.

\section{REFERENCES}

[1] Decreto Interministeriale 211 del 7 ottobre 2010 - Indicazioni Nazionali per i Licei.

[2] Regolamenti di Riordino dei Licei, degli istituti tecnici e degli istituti professionali emanati dal Presidente della Repubblica in data 15 marzo 2010.

[3] W. Maraschini, "Intervento alla Tavola rotonda- Convegno UMI, Cetraro ", XXIX Convegno CIIM, 2010. Retrieved from http://www.umi-ciim.it/wp-content/uploads/2013/12/Maraschini.pdf.

[4] I. Toth, "No! Libertà e verità creazione e negazione. Palinsesto di parole e immagini", Bompiani, Milano, 2003.

[5] I. Toth, "La geometria non euclidea come atto di creazione", video taken from Enciclopedia Multimediale delle Scienze Filosofiche, Università "Federico II", Napoli, 1991. Retrieved from http://www.conoscenza.rai.it/site/it-

IT/?ContentID=383\&Guid=85321985043d44b5b46c9562ccc84d79\#.

[6] Circolare ministeriale n. 615 del 27 settembre 1996 prot. n. 2704 - Piano nazionale per l'introduzione dell'Informatica nelle scuole secondarie superiori Indicazioni programmatiche relative all'insegnamento della matematica nel triennio del liceo ginnasio e del liceo scientifico e nel secondo biennio dell'istituto magistrale.

[7] C. Di Foggia, T. Olivello, R. Prosperi, A. Rotunno, "Una presentazione didattica delle geometrie non euclidee", Mathesis, 2004.

[8] T. Olivello, N. Tedesco, A. Trampetti, "Città, norme e strade: una proposta didattica per costruire in maniera alternativa sistemi assiomatici", La matematica e la sua didattica, no. 01, Pitagora Editrice, Bologna.

[9] A. Arcara, "Le geometrie non euclidee: introduzione e modelli", Final report for Scuola Interuniversitaria Lombarda di Specializzazione, 2005. Retrieved from https://www.matematicamente.it/tesi-didattica/Arcara-geometrie_non_euclidee.pdf

[10] E. Agazzi, D. Palladino, "Le geometrie non euclidee e i fondamenti della geometria", Analisi e Sintesi, La Scuola, 1998.

[11] S. Benvenuti, "Geometrie non euclidee", Alpha Test, Gli Spilli, 2008.

[12] H. S. M. Coxeter, "Geometria non-euclidea", Le scienze matematiche, Unione Matematica Italiana, Zanichelli 1973.

[13] "Le geometrie non euclidee" (web site). Retrieved from: progettomatematica.dm.unibo.it/NonEuclidea/

[14] J. Castellanos, J. Dan Austin, E. Darnell, M. Estrada, "An Empirical Exploration of the Poincaré Model for Hyperbolic Geometry", Mathematics and Computer Education, I Volume, 27 Issue, pp.51-pp.68, 1993. 
[15] Panagiota Kotarinou, Charoula Stathopoulou, "ICT and Liminal Performative Space for Hyperbolic Geometry's Teaching", Chapter in Mathematics and Technology, pp.75-pp-98 Springer, 2017.

[16] D. Damcke, T. Dray, M. Fung, D. Hart, L. Riverstone, "Using non-Euclidean Geometry to teach Euclidean Geometry to K-12 teachers", 2008. Retrieved from http://math.oregonstate.edu/ tevian/OMLI/Using.pdf 